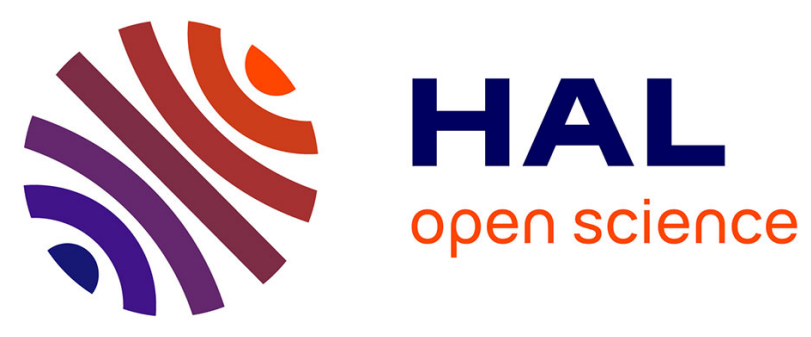

\title{
Identification of a peripheral blood gene signature predicting aortic valve calcification
}

Donal Macgrogan, Beatriz Martínez-Poveda, Jean-Pierre Desvignes, Leticia Fernandez-Friera, Manuel José Gomez, Eduardo Gil Vilariño, Sergio Callejas Alejano, Pablo García-Pavía, Jorge Solis, Joaquín Lucena, et al.

\section{To cite this version:}

Donal Macgrogan, Beatriz Martínez-Poveda, Jean-Pierre Desvignes, Leticia Fernandez-Friera, Manuel José Gomez, et al.. Identification of a peripheral blood gene signature predicting aortic valve calcification. Physiological Genomics, 2020, 52 (12), pp.563-574. 10.1152/physiolgenomics.00034.2020 . hal-02965646

\section{HAL Id: hal-02965646 \\ https: / / hal-amu.archives-ouvertes.fr/hal-02965646}

Submitted on 28 Apr 2021

HAL is a multi-disciplinary open access archive for the deposit and dissemination of scientific research documents, whether they are published or not. The documents may come from teaching and research institutions in France or abroad, or from public or private research centers.
L'archive ouverte pluridisciplinaire HAL, est destinée au dépôt et à la diffusion de documents scientifiques de niveau recherche, publiés ou non, émanant des établissements d'enseignement et de recherche français ou étrangers, des laboratoires publics ou privés. 
1 MS PG-00034-2020 revised: Identification of a peripheral blood gene signature predicting aortic valve calcification

Running title: A gene signature predicting aortic valve calcification

4

Donal MacGrogan ${ }^{1,2, *}$, Beatriz Martínez Poveda ${ }^{1,2}$, Jean-Pierre Desvignes ${ }^{3}$, Leticia Fernandez-Friera ${ }^{2,4,5}$, Manuel José Gomez ${ }^{6}$, Eduardo Gil Vilariño ${ }^{7}$, Sergio Callejas Alejano ${ }^{7}$, Pablo Garcia-Pavia ${ }^{2,8,9}$, Jorge Solis $^{10}$, Joaquín Lucena ${ }^{11}$, David Salgado ${ }^{3}$, Gwenaelle Collod-Béroud ${ }^{3}$, Emilie Faure ${ }^{3}$, Alexis Théron ${ }^{12}$, Julia Torrents ${ }^{13}$, Jean-François Avierinos ${ }^{3,12}$, Lorena Montes ${ }^{14}$, Ana Dopazo ${ }^{7}$, Valentín Fuster ${ }^{15,16}$, Borja 


\section{ABSTRACT}

48

Calcific aortic valve disease (CAVD) is a significant cause of illness and death worldwide. Identification of early predictive markers could help optimize patient management. RNAsequencing was carried out on human fetal aortic valves at gestational weeks 9, 13, and 22, and on a case-control study with adult non-calcified and calcified bicuspid and tricuspid aortic valves. In dimension reduction and clustering analyses, diseased valves tended to cluster with fetal valves at week 9 rather than normal adult valves, suggesting that part of the disease program might be due to re-iterated developmental processes. The analysis of groups of coregulated genes revealed predominant immune-metabolic signatures, including innate and adaptive immune responses involving lymphocyte $\mathrm{T}$ cell metabolic adaptation. Cytokine and chemokine signaling, cell migration, and proliferation were all increased in CAVD, whereas oxidative phosphorylation and protein translation were decreased. Discrete immune-metabolic gene signatures were present at fetal stages and increased in adult controls, suggesting that these processes intensify throughout life and heighten in disease. Cellular stress-response and neurodegeneration gene signatures were aberrantly expressed in CAVD, pointing to a mechanistic link between chronic inflammation and biological ageing. Comparison of the valve RNA-sequencing dataset with a case-control study of whole blood transcriptomes from asymptomatic individuals with early aortic valve calcification identified a highly predictive gene signature of CAVD and of moderate aortic valve calcification in overtly healthy individuals. These data deepen and broaden our understanding of the molecular basis of CAVD and identify a peripheral blood gene signature for the early detection of aortic valve calcification.

\section{Keywords}

Human fetal valve; CAVD; inflammation; peripheral blood biomarker; gene signature 


\section{INTRODUCTION}

73 Calcific aortic valve disease (CAVD) is caused by calcium build-up of the aortic valve leaflet

74 and may be associated with hemodynamic obstruction, leading to different degrees of aortic

75 stenosis (AS). Untreated severe AS leads to heart failure, with valve replacement remaining the

76 only available treatment. Severe AS is preceded by aortic sclerosis, a subclinical form of CAVD

77 that develops over many years without hemodynamic consequence (6). The prevalence of aortic

78 valve disorders increases with age, with $25 \%$ of individuals $>65$ years with aortic sclerosis

79 progressing to AS at a rate of $2 \%$ per year (4). Other than age, the principal CAVD risk factor for

80 AS is a congenitally malformed bicuspid aortic valve (BAV), which is present in $0.5-2 \%$ of the

81 general population (31). Patients with AS and a BAV are a decade younger at diagnosis than

82 those with normal tricuspid aortic valve (TAV) morphology (2). Premature leaflet thickening and

83 calcification in BAV patients may be caused by abnormal shear stress hemodynamics.

84 CAVD shares mechanistic features with atherosclerosis (6). The current accepted model

85 implicates initial lipid oxidation, inflammatory infiltration by T-lymphocytes and macrophages,

86 cytokine and pro-fibrotic signaling, and altered extracellular matrix (ECM) secretion, leading to

87 ectopic bone formation and tissue mineralization $(11,22)$. The factors predicting transition from

88 the "at risk" status to aortic sclerosis and subsequently to severe AS, are not known (25).

89 Transcriptional profiling of CAVD has helped to identify hundreds of genes differentially

90 expressed between calcified and normal valves (8). Multi-omics approaches combining

91 transcriptomics and proteomics have provided a high-resolution expression atlas and established

92 potential drivers of disease progression (30).

93 Despite this progress, thus far no medical therapy has been proven to be effective in 94 preventing progression to severe disease, with randomized clinical trials demonstrating the lack

95 of effect of statins on disease progression (36). It has been suggested that statin treatment would

96 have to begin at an earlier disease stage to have a beneficial effect. Current imaging modalities,

97 especially echocardiography, remain the gold standard for diagnosis of valve disease but are 
mainly performed when symptoms appear. Non-contrast computed tomography may have significant predictive value in both asymptomatic and symptomatic patients, but remains a source of radiation exposure and may not be available in centers with limited resources. Biomarkers may complement imaging modalities to detect early changes, especially early calcification. The ideal biomarker would predict disease as well as improve prognosis, subclinical disease management, and risk stratification and reduce disease-associated morbidity and mortality. Already identified potential biomarkers include markers of myocardial injury, cardiac mechanical stretch, inflammation, and hemostasis imbalance; however, with the exception of lipoprotein(a), these are not specific to CAVD or have yet to be validated in large randomized studies (32).

The aortic valve develops from rudimentary endocardial cushions that are subsequently refined into thin leaflets well into postnatal life. This process is dependent on complex cell-ECM interactions and is modulated by hemodynamics, and its spatio-temporal dysregulation results in malformations and maladaptive remodeling (10). Several lines of evidence indicate that valve disease at any age is accompanied by some underlying structural defect (29), suggesting that latent CAVD has a developmental origin. Moreover, developmental pathways may also be reactivated during tissue repair processes in the disease setting. For example, molecular mechanisms controlling heart valve cell differentiation are shared with cartilage, tendon, and bone development (reviewed in (15), and are reactivated during endochondral ossification and mature lamellar bone formation taking place in end-stage aortic valve disease (18). Remarkably, only end-stage CAVD has been described at the molecular level, whereas the molecular underpinnings of subclinical disease are poorly understood, due in part to difficulties in accessing biological material from early lesions.

To gain mechanistic insight, we performed an RNA-seq analysis of human fetal and adult control and calcified bicuspid (cBAV) and tricuspid valves (cTAV). K-means clustering was used to identify dynamically regulated processes taking place simultaneously at crucial stages of 
124 human valve development and in diseased valves. The prevailing gene signatures were 125 associated with immune-metabolic processes and heretofore uncharacterized pathways linked to 126 cellular stress and ageing. A search for genes shared between our gene expression datasets and 127 datasets generated from asymptomatic subjects from the Progression of Early Subclinical 128 Atherosclerosis (PESA) cohort (5) with aortic valve calcification and without AS identified 129 marker genes in peripheral blood of asymptomatic individuals. When combined in quantitative 130 RT-PCR assays, these marker genes showed good sensitivity and specificity for predicting 131 CAVD at a subclinical stage. 


\section{RESULTS}

\section{Diseased valves are transcriptionally more similar to developing valves than to controls}

Genome-wide transcriptome profiling was carried out on normal adult control valves

$135(\mathrm{n}=8)$ and severely stenotic calcified BAV (cBAV; $\mathrm{n}=5)$ and TAV (cTAV; $\mathrm{n}=7)$ valves, as well

136 as on fetal aortic valves at gestational weeks $9(n=4), 12-13,(n=3)$ and $22(n=2)$ (Supplementary

137 Table S1, see https://figshare.com/s/4357d56025cb3380a0df). These developmental timepoints

138 correspond to mouse embryonic days (E) 13.5-17.5 (13), during which valves are remodeled by

139 morphogenic processes leading to thinning, elongation, and formation of multi-laminar valve

140 structure. To integrate data across studies and generalize our findings, we conducted a meta-

141 analysis with publicly available expression data from normal adult control ( $\mathrm{n}=8)$, cBAVs $(\mathrm{n}=10)$,

142 and cTAVs ( $\mathrm{n}=9$ ) valves (8) (Figure 1A). After correcting for batch effects, Principal Component

143 Analysis (PCA) indicated that sample differences did not correlate with batch origin

144 (Supplementary Fig. S1A), but rather depended on disease or developmental stages (Figure 1B).

145 The first of the PCA components (PC1) separated developing and adult valve samples according

146 to disease severity, ranging from cTAV (right hand side) to controls (left hand side).

147 Interestingly, fetal valves tended to cluster with diseased adult valves. There were two control

148 samples (both from heart transplants) and one sample of embryonic samples at 9 weeks showing

149 more heterogeneity than the rest according to the second component (y-axis) but still the range of

150 the axis (-0.6 to 0.4$)$ is very small, confirming the reproducibility of the samples in terms of

151 global transcriptome. In the second PCA component (PC2), the adult control and disease samples

152 were separated from fetal weeks 9 and 13 samples (Figure 1B). Therefore, and considering that

153 the first PCA dimension explains $15.24 \%$ of the variance, while the second dimension explains

154 only $7.73 \%$, adult disease samples appeared to be more closely related to fetal than to control

155 samples, suggesting that some of the disease pathogenesis can be ascribed to the recapitulation of

156 developmental processes. 

under the same branch with embryonic samples at 9 and 13 weeks.

\section{Clustering identifies dynamic gene expression across disease and developmental states}

163 Differential expression analysis was initially carried out between each pair of conditions of

164 interest (Supplementary Table S2, see https://figshare.com/s/4357d56025cb3380a0df

$165 ; 9$ pairwise comparisons). However, pair-wise comparisons were not adequate to explore 166 dynamic and coordinated gene expression profiles across different disease and developmental 167 states. K-means analysis computing $\mathrm{n}=50$ yielded dynamic expression profiles across the

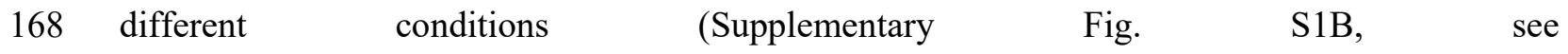

169 https://figshare.com/s/d9a68e0d6faa67e4c6c3). Co-expressed genes (ie. genes undergoing 170 similar disease/stage status expression changes) likely participate in the same process, and genes 171 that function in the same process are regulated coordinately (20). There were 6 broad

172 combinations of expression patterns: 1) genes down-regulated in developing valves and up-

173 regulated in control adult valves (e.g. C34); 2) genes down-regulated in control and up-regulated 174 in diseased valves (e.g. C7); 3) genes up-regulated in developing valves, down-regulated in adult 175 control valves, and up-regulated in diseased valves (e.g. C45); 4) genes up-regulated in 176 developing valves and down-regulated in adult control and disease valves (e.g. C37); 5) genes 177 up-regulated in adult control valves and down-regulated in diseased valves (e.g. C16); and 6) 178 genes down-regulated in developing valves, up-regulated in adult control valves, and down179 regulated in diseased valves (e.g. C40) (Supplementary Fig. S1B, see 180 https://figshare.com/s/d9a68e0d6faa67e4c6c33). Thus, part of the disease process involves a gene 181 signature present during fetal valve development. 
183 Comparative functional analysis identifies multiple interconnected pathways

184 Functional enrichment analysis of clusters with IPA identified significant associations to

185 canonical pathways in 28 clusters (Figure 2). Subsequent unsupervised hierarchical clustering of

186 clusters, based on the fraction of shared canonical pathways, identified 6 metaclusters (MC),

187 termed MC1-MC6 (Figure 2). Cluster 10 did not overlap with the others in terms of functional

188 enrichment, and was not further considered. Generally, genes highly expressed in adult disease

189 showed low expression at gestational week 13 and vice-versa; however, there were exceptions

190 (clusters 9, 12, 23, and 45; Figure 2). Although metaclusters were defined as non-overlapping

191 collections of gene clusters, enriched functions could be shared between several meta-clusters.

192 For example, canonical pathways associated to clusters 46 and 32 were shared by MC1 and

193 MC2; those associated to cluster 12 were shared by MC2, MC3, MC4, and MC6; those to

194 clusters 12 to 45 were shared by MC2, MC3, and MC4; and those canonical pathways enriched

195 in clusters 5 and 45 were shared by MC4 and MC6 (Figure 2). Therefore, the biological

196 functions represented in the different MCs are interconnected.

Innate and adaptive immune processes are present in valve disease and development

199 The collections of genes associated to metaclusters MC1-MC6 were reanalysed with IPA.

200 Enriched canonical pathways associated to each MC are summarized in Figure 3. MC3 was

201 associated to 168 pathways related to innate-adaptive immune processes (Supplementary Fig. S2,

202 see https://figshare.com/s/d9a68e0d6faa67e4c6c3). The top 5 pathways were Th1 and Th2

203 Activation Pathway, TREM1 Signaling, Communication between Innate and Adaptive Immune

204 Cells, Phagosome Formation and Dendritic Cell Maturation (Figure 3; Supplementary Fig. S3,

205 see https://figshare.com/s/d9a68e0d6faa67e4c6c3; $\quad$ Supplementary $\quad$ Table $\quad$ S3, see

206 https://figshare.com/s/4357d56025cb3380a0df). The remaining 163 enriched biological

207 functions were also involved in critical immune-inflammatory cellular processes (Supplementary

208 Fig. S2, see https://figshare.com/s/d9a68e0d6faa67e4c6c3; Supplementary Table S3, see 
https://figshare.com/s/4357d56025cb3380a0df). Among the nine clusters combined into MC3,

210 five of them had expression profiles characterised by increased gene expression in all adult

211 samples, both control and CAVD (clusters 22, 9, 34, 36 and 2), whereas four clusters had

212 increased expression in CAVD only (clusters 49, 44, 7, and 35; Figure 3). Discrete immune cell

213 gene signatures were present at week 9 (clusters 44 and 35), week 13 (cluster 9), and week 22

214 (clusters 49 and 22) of development (Figure 3), but involved relatively fewer pathways than

215 those found in control or calcified adult valves. The presence of immune and pro-inflammatory

216 gene signatures during fetal development and in apparently healthy valves is consistent with the

217 progressive nature of the calcified valve disease pathology.

\section{Cell migration and DNA replicative pathways are re-activated in CAVD}

220 Metaclusters MC4 and MC6 were enriched in genes belonging to 202 and 23 IPA pathways, 221 respectively (Supplementary $\quad$ Fig. $\quad$ S4 $\quad$ and S6A, see

222 https://figshare.com/s/d9a68e0d6faa67e4c6c3; $\quad$ Supplementary $\quad$ Table S3, see

223 https://figshare.com/s/4357d56025cb3380a0df). In general, genes associated to these clusters

224 have increased expression during development (notably at week 13), decreased in adulthood and 225 re-activated in CAVD. Therefore, of the 7 clusters associated with these two MCs, six were 226 associated with more elevated gene expression in the diseased state (clusters 25, 23, 5, 45, 17, 227 and 21), whereas only one was associated with lower expression (cluster 40; Figure 3). The top 228 five pathways in MC4 were Actin Cytoskeleton Signaling, Molecular Mechanisms of Cancer, 229 Axonal Guidance Signaling, Epithelial Adherens Junction Signaling and Integrin Signaling 230 (Figure 3, Supplementary Fig. S5, Supplementary Table S3, see 231 https://figshare.com/s/4357d56025cb3380a0df). The remaining 197 IPA pathways, represented 232 components of fundamental cell and developmental signaling pathways (Supplementary Fig. S4, 233 see https://figshare.com/s/d9a68e0d6faa67e4c6c3; Supplementary $\quad$ Table S3, see 234 https://figshare.com/s/4357d56025cb3380a0df). The top five pathways in MC6 were Role of 
235 CHK Proteins in Cell Cycle Checkpoint Control, Sumoylation Pathway, Cell Cycle Control of 236 Chromosomal Replication, Cell Cycle: G2/M DNA Damage Checkpoint Regulation, Role of 237 BRCA1 in DNA Damage Response (Figure 3, Supplementary Fig. S6B, see 238 https://figshare.com/s/d9a68e0d6faa67e4c6c3, Supplementary $\quad$ Table S3, see 239 https://figshare.com/s/4357d56025cb3380a0df). The remaining 18 IPA pathways were involved 240 in similar processes (Supplementary $\quad$ Fig. S6A, see

241 https://figshare.com/s/d9a68e0d6faa67e4c6c3; $\quad$ Supplementary $\quad$ Table $\quad$ S3, see 242 https://figshare.com/s/4357d56025cb3380a0df). Cellular proliferation and migration may be 243 part of the ongoing immune-inflammatory response associated with processes represented in 244 MC3.

246 Decreased OXPHOS and ribosomal biogenesis in CAVD

247 Genes in MC2 were significantly associated to 83 IPA pathways (Supplementary Fig. S7, see 248 https://figshare.com/s/d9a68e0d6faa67e4c6c3; Supplementary $\quad$ Table $\quad$ S3, see 249 https://figshare.com/s/4357d56025cb3380a0df), the top 5 of which were Mitochondrial 250 Dysfunction, EIF2 Signaling, Oxidative Phosphorylation, Sirtuin Signaling Pathway 251 (Supplementary Fig. S8A-C), Regulation of eIF4 and p70S6K Signaling (Supplementary Fig. 252 S8D,E see https://figshare.com/s/d9a68e0d6faa67e4c6c3; Supplementary Table S3, see 253 https://figshare.com/s/4357d56025cb3380a0df). The remaining 78 pathways in MC2 were also 254 implicated in energy production and protein metabolism (Supplementary Fig. S7, see 255 https://figshare.com/s/d9a68ed6faa67e4c6c3; $; \quad$ Supplementary Table S3, see 256 https://figshare.com/s/4357d56025cb3380a0df), showing strong enrichment in genes for nuclear 257 encoded subunits of the electron transport chain complexes and ribosomal biogenesis 258 (Supplementary Fig. S8A-C, see https://figshare.com/s/d9a68e0d6faa67e4c6c3; Supplementary 259 Table S3, see https://figshare.com/s/4357d56025cb3380a0df). Other gene signatures were AMP260 activated protein kinase (AMPK), ER stress (Unfolded protein response), oxidative stress 
261 response (NRF2-mediated Oxidative Stress Response), cellular longevity (Telomerase

262 Signaling), and neurodegenerative disease (Huntington's Disease Signaling; Supplementary Fig.

263 S7, see https://figshare.com/s/d9a68e0d6faa67e4c6c3; Supplementary Table S3, see

264 https://figshare.com/s/4357d56025cb3380a0df), suggesting the modulation of cellular stress

265 responses, and age-related disease gene signatures. Of the nine clusters in MC2, six had, on

266 average, decreased expression in disease conditions (clusters 24, 31, 32, 16, 41, and 1), while

267 one had increased expression in the diseased state (cluster 12) and three were unchanged

268 (clusters 31, 26, and 37; Figure 3). In general, MC2 transcripts had peak expression at week 13,

269 with lower expression at the other developmental stages (Figure 3), suggesting that metabolic

270 processes are dynamically regulated during aortic valve development. Overall, this analysis

271 indicates that CAVD is associated with decreased OXPHOS metabolism and altered proteostasis.

272

273 Stress response gene signatures are altered in CAVD

274 MC1 and MC5 did not overlap significantly with any of the other MCs but included similar

275 biological processes. Genes in MC1 were associated to 7 pathways (Figure 3; Supplementary

276 Fig. S9A, see https://figshare.com/s/d9a68e0d6faa67e4c6c3; Supplementary Table S3, see

277 https://figshare.com/s/4357d56025cb3380a0df), the top 5 being Sphingosine-1-phosphate

278 Signaling, Endocannabinoid Developing Neuron Pathway, Amyloid Processing, Huntington's

279 Disease Signaling, Dermatan Sulfate Degradation (Figure 3; Supplementary Fig. S9B, see

280 https://figshare.com/s/d9a68e0d6faa67e4c6c3; $\quad$ Supplementary $\quad$ Table $\quad$ S3, see

281 https://figshare.com/s/4357d56025cb3380a0df). The expression of MC1 genes was relatively

282 increased in adult controls and decreased in CAVD (Figure 3). MC5 was enriched in a single

283 pathway, Unfolded protein response (Figure 3; Supplementary Fig. S9C, see

284 https://figshare.com/s/d9a68e0d6faa67e4c6c3; $\quad$ Supplementary $\quad$ Table $\quad$ S3, see

285 https://figshare.com/s/4357d56025cb3380a0df). MC5 genes were expressed at decreased levels

286 in controls and increased in CAVD (Figure 3). These data suggest that chronic immune- 
287 inflammatory processes are linked to cellular stress and age-related disease in calcific aortic 288 valve pathology.

291 Identification of circulating biomarkers predicting CAVD

292 To identify potential biomarkers useful for predicting CAVD, a sub-set of genes from the aortic

293 valve study was compared with whole blood RNA-Seq data from asymptomatic individuals with 294 aortic valve calcification, detected by non-contrast computed tomography in the Progression of 295 Early Subclinical Atherosclerosis (PESA) study (5). PESA participants undergo simultaneous 296 cardiac calcium scoring and blood sampling. Out of the 455 PESA participants for which RNA297 seq data was available in visit 2 (ie. three years after recruitment), 59 had evidence of valve 298 calcification and 52 of theses had sufficient integrity for qPCR analysis. None of them had 299 valvular stenosis/regurgitation, evidencing an early stage of the disease. Their transcriptome was 300 compared to the transcriptome of PESA individuals without valvular calcification $(\mathrm{n}=395)$. From 301 the whole set of genes identified as differentially expressed ( $<<0.05$, Supplementary Table S5, 302 see https://figshare.com/s/4357d56025cb3380a0df), six were selected that showed a disease303 specific pattern in the RNA-seq data of human aortic valves (see Methods for selection criteria;

304 Supplementary Fig. S1, see https://figshare.com/s/d9a68e0d6faa67e4c6c3). Five of these genes 305 were related to adaptive innate and adaptive responses (CD28, ITK, PAG1, NLRC5, and ASCC3) 306 and a sixth was related to the WNT pathway (LINS1) (Supplementary Table S6, see 307 https://figshare.com/s/4357d56025cb3380a0df). The expression of the six genes was measured in 308 whole blood (WB) on 104 PESA participants [52 cases: asymptomatic individuals with 309 calcification of the aortic valve (PESA-SD); 52 controls: individuals without calcification 310 (PESA-CTL)] and 12 patients with CAVD recruited at Monteprincipe Hospital (WB-CAVD) by 311 qRT-PCR and correlated with the degree of aortic valve calcification (Figure 4A, Supplementary 312 Table S4, see https://figshare.com/s/4357d56025cb3380a0df). Considering all conditions, the 6 
313 genes showed some degree of correlation when considered individually $(P \leq 0.27$; Figure 4A,

314 Supplementary Table S7, see https://figshare.com/s/4357d56025cb3380a0df). Given that,

315 NLRC5 and PAG1 were also associated with total calcification of the coronaries (Supplementary

316 Fig. S10A, see https://figshare.com/s/d9a68ed6faa67e4c6c3, Supplementary Table S7, see

317 https://figshare.com/s/4357d56025cb3380a0df) and were therefore excluded as potential specific

318 markers of aortic valve calcification.

319 To determine the utility of ASCC3, ITK, CD28 and LINS1 as potential markers of valve 320 calcification, a ROC curve was built to represent the predictive value of the model to classify 321 calcific disease patients (WB-CAVD) vs PESA-SD individuals and vs PESA-CTL, yielding 322 areas under the curve (AUCs) of 0.97 and 0.91 respectively (Figure 4B). An additional model 323 was built to assess the power of the gene signature to distinguish between individuals without 324 calcification (PESA-CTL) from those with subclinical disease (PESA-SD; AUC=0.71).

325 This model was then validated in whole blood samples of an independent cohort of 326 CAVD patients ( $\mathrm{n}=16)$, together with samples from 25 of the 52 PESA-SD individuals and 35 of 327 the 52 PESA-CTL individuals taken three years after visit 2 (Figure 4C). The model achieved 328 AUCs of 0.90 and 0.88 to discriminate CAVD patients from PESA-SD and PESA-CTL 329 individuals, respectively. The AUC of the model to distinguish PESA-SD from PESA-CTL was 330 again 0.71 (Figure 4C). Thus, our model identifies a gene expression signature in blood that is 331 highly predictive of valve disease and of aortic valve calcification levels in otherwise healthy 332 individuals. 
335 This study provides, for the first time, a comprehensive molecular profile of shared and unique

336 cellular activities taking place in aortic valve development and CAVD. The transcriptome of

337 adult disease samples appeared to be more closely related to fetal week 9 than to control samples

338 or weeks 13 and 22 , suggesting that some of the disease pathogenesis might be ascribed to the

339 recapitulation of processes taking place in early development. The overall cBAV and cTAV gene

340 expression profiles were essentially identical, confirming previous findings suggesting that valve

341 calcification depends on the same biological pathways, regardless of valve morphology $(8,26)$.

342 Therefore, the mechanisms leading to BAV formation are independent of those leading to BAV

343 calcification; in other words, the cause of the embryonic defect is distinct from the resulting

344 calcification process.

345 Functional analysis provided compelling evidence that innate and adaptive immune

346 interactions take place in the aortic valve throughout life (Supplementary Fig. S10B 13, see

347 https://figshare.com/s/d9a68e0d6faa67e4c6c3). The fetal immune cell gene signature in MC3

348 was notably simpler than in adult control or CAVD samples, with fewer immune cell gene

349 expression signatures present at gestational weeks 9 (3/9 clusters), 13 (1/9 clusters), or 22 (3/9

350 clusters) than in adult controls (5/9 clusters) or in CAVD (9/9 clusters), suggesting that aortic

351 valve immune cell enrichment in the aortic valve increases over time. Moreover, the presence of

352 only one immune cell gene signature at 13 weeks (cluster 9), compared with three at 9 and 22

353 weeks of gestation, implies that the immune landscape is dynamically regulated in developing

354 valves. These observations are consistent with previous findings of multiphasic changes in shear

355 stress-response, cell-ECM interaction and adaptive immunity pathways from week 14 to week 22

356 of human aortic valve development (7).

357 The predominant $\mathrm{T}$ cell gene signature in the RNA-seq dataset strengthens the notion that

358 unresolved immune-inflammatory response is a major CAVD component (17). This association

359 is well established, with early immunohistological findings indicating the presence of $\mathrm{T}$ 
360 lymphocytes in immune infiltrates $(23,24)$. More recent studies have revealed the existence of T

361 cell infiltration in BAV and TAV calcific disease, consisting of non-specific chemokine driven

362 polyclonal $\mathrm{T}$ cell recruitment, and memory-effector $\mathrm{CD} 8+\mathrm{T}$ cell clonal expansions $(34,35)$,

363 supporting the interpretation of an antigen-induced immune response. Moreover, this antigen-

364 driven response involved circulating expanded CD8+ and, to a lesser extent, CD4+ T cell clones

365 between circulating blood and valve tissues suggesting a systemic disease (34). The gene

366 expression signatures of several other immune-competent cells, including B-lymphocytes,

367 macrophages, and dendritic cells were also present, confirming previous characterization of

368 cellular infiltrates in CAVD (33). Together with a body of literature suggesting a tight

369 inflammatory cell-valve interstitial cell interaction in $\operatorname{CAVD}(11,17)$, our data are consistent

370 with the notion that unresolved inflammation ultimately drives myo-fibroblast and osteoblast-

371 like differentiation and leads to heterotopic bone formation. Therefore, targeted therapeutic

372 immunosuppression of calcification might be approachable by better characterizing the various

373 infiltrating immune-cell subtypes.

374 Functional analysis revealed that OXPHOS metabolism was decreased in CAVD, with

375 coordinated expression reduction of multiple nuclear genes encoding subunits of mitochondrial

376 electron transport chain complexes I, III, IV and V. These changes might be part of ongoing

377 adaptive metabolic reprogramming during $\mathrm{T}$ lymphocyte activation (27), when upon

378 encountering an antigen, $\mathrm{T}$ cells proliferate and acquire new functions, switching energy

379 metabolism from OXPHOS to glycolysis. However, both increased and decreased expression of

380 glycolytic pathway components were present, suggesting that decreased OXPHOS metabolism

381 cannot be ascribed solely to T cell activation. Moreover, adult aortic valves showed markedly

382 reduced transcript levels of multiple subunits of the small and large protein ribosomal complexes

383 (RPS and RPL respectively) and eukaryotic translation initiation factors (EIF1-4), possibly

384 reflecting co-ordinate dysregulation of OXPHOS activity and ribosomal biogenesis (19). This

385 occurred in uncalcified adult valves as well (clusters 31, 37, and 12), suggesting declining 
386 function with age. In contrast signatures for cell death and apoptosis (for example in MC3) and,

387 energy-demanding cell processes such as proliferation, DNA repair, and cell movement (in MC4

388 and MC6) were increased, possibly reflecting cellular heterogeneity within the calcified valve.

389 Decreased mitochondrial metabolism and protein translation may be a manifestation of

390 altered physiological stress responses that occur during ageing (30), which is also related to the

391 inability to maintain adaptability to stress and re-establish homeostasis in response to

392 environmental perturbations. Thus, multiple stress-response gene signatures were present in

393 MC2 and to a lesser extent in MC1 and MC5 (Supplementary Fig. S10B, see

394 https://figshare.com/s/d9a68e0d6faa67e4c6c33). These included SIRT, a highly conserved family

395 of NAD-dependent deacetylases, linking transcriptional regulation to mitochondrial function,

396 stress resistance, metabolism, cell survival and organismal longevity (3) and mTOR (Mammalian

397 target of rapamycin), a nutrient sensing system which regulates protein synthesis, to promote cell

398 growth and proliferation (19). Moreover, deficiency of the unfolded protein response (UPR)

399 pathway, which is activated by misfolding of newly synthesized proteins as part of the ER stress

400 response (9) contributes to inflammation, disease and ageing. Gene signatures including amyloid

401 processing and Huntington's disease signaling were also featured implying shared calcific valve

402 and neurodegenerative disease mechanisms (Supplementary Fig. S10B, see

403 https://figshare.com/s/d9a68e0d6faa67e4c6c3). Therefore, chronic inflammation drives

404 metabolic stress and biological ageing in CAVD.

405 Given the phenomenon of cellular trafficking of $\mathrm{T}$ cells between the diseased valve

406 tissue, and circulating blood (34), we reasoned that aortic valve and circulating blood from

407 CAVD patients share common genes that would be absent in subclinical individuals. In the

408 PESA study we identified a subpopulation of asymptomatic individuals in this cohort with $>1$

409 valve calcification detected by imaging. Therefore, we searched for circulating biomarkers by

410 comparing the CAVD transcriptomes and whole blood from datasets of the subclinical PESA

411 study population, and identified six differentially expressed genes, four of which were found to 
412 predict subclinical and overt valve disease. Therefore ASCC3, ITK, CD28, and LINS were 413 specific to aortic valve calcification, whereas PAG1 and NLRC5 were also associated with 414 coronary artery calcification, suggesting that valve and vascular calcification proceed by shared 415 and separate mechanisms. These mechanisms have yet to be examined, but noteworthy is that 416 five of six genes belong to T cell activation and NF-kB pathways. This gene signature could be 417 used in combination with high-resolution imaging for the early diagnosis of valve calcification. 418 Study Limitations. A limitation of our study is the low sample numbers especially for 419 week 22 samples $(\mathrm{n}=2)$. Investigational studies involving human fetal issue are limited by ethical 420 and legal considerations on medical abortion. Nevertheless, our bioinformatics analysis showed 421 that the week 22 samples clustered together and separated well from the other groups by PCA 422 and we are confident of the robustness of the results involving this group. Moreover, differential 423 expression analysis was performed using limma, a statistical method developed ad-hoc for RNA424 seq data with low sample size (28).

425

426

427

428 
430 Study population and aortic valve collection.

431 Fetuses. Aortic valves from human fetuses were obtained from electively- (weeks 9; n=4 and 432 week $12 / 13 ; n=3$ ) or medically (week $22 ; n=2$ ) -terminated pregnancies after written informed 433 consent in concordance with French legislation (PFS14-011) and prior protocol approval from 434 the "Agence de la biomédecine" (Supplementary Table S1, see 435 https://figshare.com/s/4357d56025cb3380a0df). Aortic valves were recognized by their 436 anatomical landmarks under the microscope and leaflets were isolated with minimal aortic wall 437 contamination Samples were immediately flash frozen in liquid nitrogen. The investigation conformed to the principles outlined in the Declaration of Helsinki.

Patients. The CAVD study population consisted of BAV $(\mathrm{n}=5)$ or TAV $(\mathrm{n}=7)$ patients referred

440 for aortic valve or aortic root surgery at Monteprincipe Hospital and Hospital Clínico San Carlos

441 (Madrid, Spain; Supplementary Table S1, see https://figshare.com/s/4357d56025cb3380a0df).

442 Patients were prospectively recruited between December 2009 and February 2013. Patients with

443 a history of rheumatic heart disease, infective endocarditis, or connective tissue disorders were 444 excluded. To determine the morphology and functional state of the aortic valve, all patients 445 underwent clinical evaluation and preoperative 2-dimensional echocardiography according to the American Heart Association and the American College of Cardiology guidelines (21). The aortic valve morphology and the presence of valvular degeneration, including the extent of 448 leaflet calcification, thickening, prolapse, and/or redundancy, were documented at surgery. 449 Calcification was graded as mild (1/3 leaflet area affected) or severe ( $2 / 3$ leaflet area affected). 450 Immediately following surgical removal, a portion of the aortic valve leaflet was placed in sterile 451 saline buffer and stored at $-80^{\circ} \mathrm{C}$ until processing. Control valve samples were obtained at 452 autopsy of individuals without cardiac problems who suffered a traumatic death $(\mathrm{n}=6)$ or at time 453 of transplantation from heart transplant receptors with normal aortic valves $(n=2)$. All patients or 454 close relatives (for autopsy controls) gave written informed consent, and the study was approved 
455 by the Health Service Ethics Committees (Comité Ético de Investigación Clínica) of participant 456 centers. The investigation conformed to the principles outlined in the Declaration of Helsinki.

457 Whole-blood samples. Genes of interest were quantified by quantitative reverse transcription458 polymerase chain reaction (qRT-PCR) in whole-blood (WB) samples from age-matched case459 control males $(n=104)$ of the Progression of Early Subclinical Atherosclerosis (PESA). 460 Subclinical cases (PESA-SD) were defined as participants with aortic-valve calcification score $461>1$, as assessed by the Agatston method (1). Controls (PESA-CTL) had no calcification of the 462 aortic valves or coronary arteries. Patients with aortic valve stenosis and moderate to severe 463 calcification (WB-CAVD; $n=12$ ) were recruited from Monteprincipe Hospital (Supplementary 464 Table S4, see https://figshare.com/s/4357d56025cb3380a0df). For validation, an independent 465 cohort of mainly severe aortic valve stenosis patients $(n=16)$ was obtained from the MRVALVE 466 study. Whole blood was collected in PAXGene tubes (BDBiosciences) and stored at $-80^{\circ} \mathrm{C}$ 467 before processing.

\section{RNA isolation}

469 For each valve, total RNA from a single aortic valve cusp was extracted using the TRIzol LS 470 (Invitrogen, Carlsbad, CA, USA) method and digested with RNase free DNase I (Invitrogen). 471 For PESA and CAVD validation total RNA was using either the PAXgene blood mRNA kit 472 (PreAnalytiX, Hombrechtikon, Switzerland) for manual isolation or the QIAsymphony PAXgene 473 blood RNA kit (PreAnalytiX) for automated isolation using a QIAsymphony SP liquid handling 474 robot (Qiagen, Venlo, Netherlands). RNA purity, concentration, and integrity were assessed by 475 Nanodrop 2000 spectrophotometry (Thermo Scientific) and automated electrophoresis in a 2100 476 Bioanalyzer (RNA6000 Nano LabChip; Agilent). Samples with a RIN $>6$ were selected for 477 reverse transcription.

\section{RNA-seq data generation}

479 RNA-seq was performed on DNase I-treated RNA samples with a RIN $>7$. RNA-seq libraries 480 were created using the TruSeq Stranded Total RNA with Ribo-Zero Gold Prep Kit (Fetal 
481 samples) or TruSeq $^{\text {TM }}$ RNA Sample Prep Kit v2 (Patient samples; Illumina, Diego, CA). Final

482 cDNA libraries were checked for quality and quantified in the 2100 Bioanalyzer (Agilent). The

483 libraries were loaded in the flow cell at $8 \mathrm{pM}$, and clusters were generated in Cbot and sequenced

484 as 50bp single-end reads on a Hiseq 2500 instrument (adult samples) or 75 base single-end reads

485 on a Genome Analyzer IIx instrument (fetal samples; Illumina, Diego, CA). Image analysis and

486 base calling were performed using RTA and CASAVA.

487 Bioinformatics analysis of RNA-Seq.

$488 \quad$ Data preprocessing

489 FASTQ files were pre-processed with cutadapt (16) to eliminate Illumina adapter remains and

490 reads were aligned to the human reference transcriptome (GRCh38.78) using RSEM 1.2.31 (14).

491 Raw data from 27 RNA-seq samples from GSE76718 were downloaded from GEO and pre-

492 processed and aligned using the same pipeline. Control samples were present in all experiments,

493 allowing for batch correction of the data using ComBat (12). Data were normalized using TMM

494 with log-ratio trimming $=0.3$ and sumTrim $=0.05$ and voom-transformed. Only genes with at least

$4951 \mathrm{cpm}$ in at least 10 samples were considered for further analysis.

496 Differential Expression Analysis

497 Differential expression between groups was performed using mixed models with eBayes, as

498 implemented in the Bioconductor limma package (28). A gene was classified as differentially

499 expressed if its Benjamini-Hochberg adjusted $P$-value was $<0.05$ in any of the contrasts under

500 study (Supplementary Table S2, see https://figshare.com/s/4357d56025cb3380a0df).

$501 \quad$ Clustering and functional analyses

502 To identify groups of genes with similar expression profiles across the six conditions under

503 consideration, k-means clustering was performed with the R ComplexHeatmap package on all

504 expressed genes (19,962 genes). The Elbow method was used to estimate the optimal number of

505 clusters, which was set as $\mathrm{k}=50$. The number of genes per cluster was between 215 and 680 .

506 Ingenuity Pathway Analysis (IPA, Qiagen) was then used to identify significant associations 
507 between canonical pathways and clusters. Twenty-eight clusters, out of the original collection of

50850 , were found to be associated to at least one canonical pathway with Benjamini-Hochberg (B-

$509 \quad \underline{H})$ adjusted $P$-value $\quad<\quad 0.05 \quad$ (Supplementary $\quad$ Table S3,

510 https://figshare.com/s/4357d56025cb3380a0df). Concordance between canonical pathway

511 profiles was then used to identify groups of clusters that could be associated to similar functions.

512 One cluster (cluster 10) did not share any enriched pathway with any other cluster and was not

513 considered further. The remaining 27 clusters were grouped into six meta-clusters, using the

514 fraction of shared pathways as distance measure. Meta-clusters contained from 332 to 3304

515 genes (Figure 2). IPA was used again to re-annotate meta-clusters (Figure 3).

\section{Data availability}

517 Data are deposited in the NCBI GEO database under accession number GSE148219.

518 qRT-PCR for validation studies in whole blood

519 Selection of candidate genes for $q R T-P C R$. We used whole blood transcriptomics data generated

520 in the PESA study to identify genes that might potentially serve as predictors of CAVD. The

521 criteria to select these candidate genes were: (i) Genes differentially expressed between

522 individuals with $(\mathrm{n}=59)$ and without $(\mathrm{n}=396)$ calcification of the aortic valve $(\mathrm{p}$-value $<=0.05)$ but

523 not differentially expressed between individuals with and without calcification in the coronaries

524 (p-value $>0.05$; Supplementary Table S5, see https://figshare.com/s/4357d56025cb3380a0df).

525 (ii) Aortic valve- specific differentially expressed genes with a top $10 \%$ correlation between their

526 expression level and the level of calcification of the aortic valves. (iii) Their expression levels in

527 the RNA-seq samples of the aortic valves were either up-regulated in disease v.s. no disease

528 samples or they were upregulated in early embryonic valve samples and in the diseased samples

529 but not in the control samples. From the 10 genes fulfilling the three criteria, 6 were selected

530 based on their presumptive function (Supplementary Table S6, see

531 https://figshare.com/s/4357d56025cb3380a0df). 
$532 q R T-P C R$. RNA samples from PESA and CAVD patients (75ng) was reverse transcribed using

533 the High Capacity cDNA Reverse Transcription kit with RNase Inhibitor (ThermoFisher

534 Scientific). Gene expression was quantified by qPCR using TaqMan probes (ThermoFisher

535 Scientific; Supplementary Table S8) in 52 case-control pairs from the PESA study (case: positive

536 calcification of the aortic valve) and in 12 CAVD patients from Monteprincipe Hospital. Each

537 reaction was performed in triplicate and the final reaction volume was set to $10 \mu 1$. qPCRs were

538 conducted in 384-well plates (Cat. no. 4344345; Applied Biosystems) using an ABI PRISM

539 7900HT Sequence Detection System (Applied Biosystems). Each well included $3 \mu 1$ (2.25 ng)

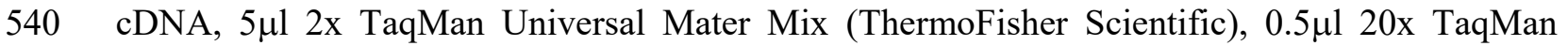

541 assay (ThermoFisher Scientific), and $1.5 \mu \mathrm{l} \mathrm{H}_{2} \mathrm{O}$. The cycling protocol was 10 min at $95^{\circ} \mathrm{C}$

542 followed by 60 cycles of $15 \mathrm{~s}$ at $95^{\circ} \mathrm{C}$ and $60 \mathrm{~s}$ at $60^{\circ} \mathrm{C}$. Baseline and threshold were set

543 automatically in SD S 2.3 (ThermoFisher Scientific) to calculate Cq values. The Cq values were

544 then imported into qBasePlus (Biogazelle, Zwijnaarde, Belgium) to calculate relative quantities

545 normalised to GAPDH. Data were scaled per sample to eliminate potential biases.

\section{Statistical analysis}

547 A linear mixed model was used to assess correlation between the expression of each gene in WB

548 and the degree of calcification in the aortic valve for each disease group (PESA-SD, WB-

549 CAVD) separately. Visualization was performed using the multiplot function and the ggplot2 R

550 package. All samples were jointly analyzed using a linear mixed model with the origin of the

551 samples as random effect. The same analysis was performed to assess the correlation of the gene

552 expression with calcification of the coronaries. Four genes which showed no correlation with the

553 amount of calcium in the coronaries were selected to build a gene expression signature using a

554 multinomial model with interaction for the prediction of the three groups of individuals with

555 different disease severity WB-CAVD>PESA-SD>PESA-CTL. To quantify the predictive power 
556 of the gene signature between different groups of individuals a logistic regression model was fit

557 to the data and the corresponding ROC curves were built using the ROCR R package.

\section{Acknowledgments}

We thank S. Bartlett for English editing. This work was supported by the Ministerio de Ciencia,

563 Innovación y Universidades from Spain, (grants SAF2016-78370-R, CB16/11/00399, CIBER

$564 \mathrm{CV}$, and RD16/0011/0021, TERCEL) and the Fundación BBVA (grant BIO14_298) and

565 Fundación La Marató TV3 (grant 20153431) to J.L.dlP; and the Fondation pour la Recherche

566 Médicale, grant from l'Institut National de la Santé et de la Recherche Médicale (grant

567 DPC20111123002) and l'Association Française contre les Myopathies (grant TRIM-RD) to

568 S.Z. The cost of this publication was supported in part with funds from the ERDF. The CNIC is

569 supported by the Ministerio de Ciencia, Innovación y Universidades (MCNU), the Instituto de

570 Salud Carlos III (ISCIII), and the Pro CNIC Foundation, and is a Severo Ochoa Center of

571 Excellence (grant SEV-2015-0505).

\section{Author contributions}

573 Donal MacGrogan, PhD: Design of work: Substantial; interpretation of data: Substantial;

574 Writing: original draft: Lead; Writing: review \& editing: Lead. final approval of the version to be 575 published.

576 Beatriz Martínez Poveda, PhD: Investigation: Lead; Methodology: Substantial; final approval of 577 the version to be published.

578 Jean-Pierre Desvignes, MD: Investigation: Supporting; final approval of the version to be 579 published.

580 Leticia Fernandez-Friera, MD, PhD: Data curation: Substantial; Formal analysis: Substantial;

581 Investigation: Supporting; final approval of the version to be published.

582 Manuel José Gomez, PhD: Formal analysis: Substantial; Visualization: Substantial; final 583 approval of the version to be published. 
584 Eduardo Gil Vilariño, BSc: Investigation: Supporting; final approval of the version to be 585 published.

586 Sergio Callejas Alejano, BSc: Investigation: Supporting; final approval of the version to be 587 published.

588 Pablo Garcia-Pavia, MD, PhD: Investigation: Supporting; final approval of the version to be 589 published.

590 Jorge Solis, MD, PhD: Investigation: Supporting; final approval of the version to be published.

591 Joaquín Lucena, MD, PhD:Investigation: Supporting; final approval of the version to be 592 published.

593 David Salgado, PhD: Data curation: supporting; Formal analysis: supporting; Investigation: 594 supporting; final approval of the version to be published.

595 Gwenaelle Collod-Béroud, PhD: Formal analysis: Supporting; Investigation: Supporting; final 596 approval of the version to be published.

597 Emilie Faure, PhD: Formal analysis: Supporting; Investigation: Supporting; final approval of the 598 version to be published.

599 Alexis Théron, MD: Investigation: Supporting; final approval of the version to be published.

600 Julia Torrents, MD: Investigation: Supporting; final approval of the version to be published.

601 Jean-François Avierinos, MD: Investigation: Supporting; final approval of the version to be 602 published.

603 Lorena Montes, MD: Investigation: Supporting; final approval of the version to be published.

604 Ana Dopazo, PhD: Investigation: Supporting; Supervision: Supporting; final approval of the 605 version to be published.

606 Valentín Fuster, MD, PhD: Investigation: Supporting; Resources: Supporting; final approval of 607 the version to be published.

608 Borja Ibañez, MD, PhD: Investigation: Supporting; Writing - review \& editing: Supporting; 609 final approval of the version to be published.

610 Fátima Sánchez-Cabo, PhD: Formal analysis: Equal; Writing - review \& editing: Supporting; 611 final approval of the version to be published.

612 Stephane Zaffran, PhD: Investigation: Supporting; Supervision: Supporting; Writing -review \& 613 editing: Supporting; final approval of the version to be published.

614 José Luis de la Pompa, PhD: Conceptualization: Lead; Funding acquisition: Lead; Project 615 administration: Lead; Resources: Lead; Supervision: Equal; Visualization: Equal; Writing 616 review \& editing: Supporting.

617 
618 Competing interests

619 The authors declare no competing interests.

620

621

622 
624 1. Agatston AS, Janowitz WR, Hildner FJ, Zusmer NR, Viamonte M, Jr., and Detrano

R. Quantification of coronary artery calcium using ultrafast computed tomography. J Am Coll Cardiol 15: 827-832, 1990.

\section{Beppu S, Suzuki S, Matsuda H, Ohmori F, Nagata S, and Miyatake K. Rapidity of} progression of aortic stenosis in patients with congenital bicuspid aortic valves. Am J Cardiol 71: 322-327, 1993.

3. Chang HC, and Guarente L. SIRT1 and other sirtuins in metabolism. Trends Endocrinol Metab 25: 138-145, 2014.

4. Coffey S, Cairns BJ, and Iung B. The modern epidemiology of heart valve disease. Heart 102: 75-85, 2016.

5. Fernandez-Ortiz A, Jimenez-Borreguero LJ, Penalvo JL, Ordovas JM, Mocoroa A, Fernandez-Friera L, Laclaustra M, Garcia L, Molina J, Mendiguren JM, Lopez-Melgar B, de Vega VM, Alonso-Farto JC, Guallar E, Sillesen H, Rudd JH, Fayad ZA, Ibanez B, Sanz G, and Fuster V. The Progression and Early detection of Subclinical Atherosclerosis (PESA) study: rationale and design. Am Heart J 166: 990-998, 2013.

6. Freeman RV, and Otto CM. Spectrum of calcific aortic valve disease: pathogenesis, disease progression, and treatment strategies. Circulation 111: 3316-3326, 2005.

7. Gottlieb Sen D, Halu A, Razzaque A, Gorham JM, Hartnett J, Seidman JG, Aikawa E, and Seidman CE. The Transcriptional Signature of Growth in Human Fetal Aortic Valve Development. Ann Thorac Surg 106: 1834-1840, 2018.

8. Guauque-Olarte S, Droit A, Tremblay-Marchand J, Gaudreault N, Kalavrouziotis D, Dagenais F, Seidman JG, Body SC, Pibarot P, Mathieu P, and Bosse Y. RNA expression profile of calcified bicuspid, tricuspid, and normal human aortic valves by RNA sequencing. Physiol Genomics 48: 749-761, 2016.

9. Hetz C. The unfolded protein response: controlling cell fate decisions under ER stress and beyond. Nat Rev Mol Cell Biol 13: 89-102, 2012.

10. Hinton RB, and Yutzey KE. Heart valve structure and function in development and disease. Annu Rev Physiol 73: 29-46, 2011.

11. Hulin A, Hego A, Lancellotti P, and Oury C. Advances in Pathophysiology of Calcific Aortic Valve Disease Propose Novel Molecular Therapeutic Targets. Front Cardiovasc Med 5: 21, 2018.

12. Johnson WE, Li C, and Rabinovic A. Adjusting batch effects in microarray expression data using empirical Bayes methods. Biostatistics 8: 118-127, 2007.

13. Krishnan A, Samtani R, Dhanantwari P, Lee E, Yamada S, Shiota K, Donofrio MT, Leatherbury L, and Lo CW. A detailed comparison of mouse and human cardiac development. Pediatr Res 76: 500-507, 2014.

14. Li B, and Dewey CN. RSEM: accurate transcript quantification from RNA-Seq data with or without a reference genome. BMC bioinformatics 12: 323, 2011.

15. Lincoln J, Lange AW, and Yutzey KE. Hearts and bones: shared regulatory mechanisms in heart valve, cartilage, tendon, and bone development. Dev Biol 294: 292-302, 2006.

16. Martin M. CUTADAPT removes adapter sequences from high-throughput sequencing reads. EMBnetjournal 17: 10-12, 2011.

17. Mathieu P, Bouchareb R, and Boulanger MC. Innate and Adaptive Immunity in Calcific Aortic Valve Disease. J Immunol Res 2015: 851945, 2015.

18. Mohler ER, 3rd, Gannon F, Reynolds C, Zimmerman R, Keane MG, and Kaplan FS. Bone formation and inflammation in cardiac valves. Circulation 103: 1522-1528, 2001. 

mTOR coordinates protein synthesis, mitochondrial activity and proliferation. Cell Cycle 14: 473-480, 2015.

674 20. Niehrs C, and Pollet N. Synexpression groups in eukaryotes. Nature 402: 483-487, 1999. 21. Nishimura RA, Otto CM, Bonow RO, Carabello BA, Erwin JP, 3rd, Guyton RA, O'Gara PT, Ruiz CE, Skubas NJ, Sorajja P, Sundt TM, 3rd, Thomas JD, Anderson JL, Halperin JL, Albert NM, Bozkurt B, Brindis RG, Creager MA, Curtis LH, DeMets D, Guyton RA, Hochman JS, Kovacs RJ, Ohman EM, Pressler SJ, Sellke FW, Shen WK, Stevenson WG, Yancy CW, American College of C, American College of Cardiology/American Heart A, and American Heart A. 2014 AHA/ACC guideline for the management of patients with valvular heart disease: a report of the American College of Cardiology/American Heart Association Task Force on Practice Guidelines. J Thorac Cardiovasc Surg 148: e1-e132, 2014.

685

686

687 22. O'Brien KD. Pathogenesis of calcific aortic valve disease: a disease process comes of age (and a good deal more). Arterioscler Thromb Vasc Biol 26: 1721-1728, 2006.

23. Olsson M, Dalsgaard CJ, Haegerstrand A, Rosenqvist M, Ryden L, and Nilsson J. Accumulation of $\mathrm{T}$ lymphocytes and expression of interleukin-2 receptors in nonrheumatic stenotic aortic valves. J Am Coll Cardiol 23: 1162-1170, 1994.

24. Otto CM, Kuusisto J, Reichenbach DD, Gown AM, and O'Brien KD. Characterization of the early lesion of 'degenerative' valvular aortic stenosis. Histological and immunohistochemical studies. Circulation 90: 844-853, 1994.

25. Otto CM, and Prendergast B. Aortic-valve stenosis--from patients at risk to severe valve obstruction. $N$ Engl J Med 371: 744-756, 2014.

26. Padang R, Bagnall RD, Tsoutsman T, Bannon PG, and Semsarian C. Comparative transcriptome profiling in human bicuspid aortic valve disease using RNA sequencing. Physiol Genomics 47: 75-87, 2015.

27. Pearce EL. Metabolism in T cell activation and differentiation. Curr Opin Immunol 22: 314-320, 2010.

28. Ritchie ME, Phipson B, Wu D, Hu Y, Law CW, Shi W, and Smyth GK. limma powers differential expression analyses for RNA-sequencing and microarray studies. Nucleic acids research 43: e47, 2015.

29. Roberts WC, and Ko JM. Frequency by decades of unicuspid, bicuspid, and tricuspid aortic valves in adults having isolated aortic valve replacement for aortic stenosis, with or without associated aortic regurgitation. Circulation 111: 920-925, 2005.

30. Schlotter F, Halu A, Goto S, Blaser MC, Body SC, Lee LH, Higashi H, DeLaughter DM, Hutcheson JD, Vyas P, Pham T, Rogers MA, Sharma A, Seidman CE, Loscalzo J, Seidman JG, Aikawa M, Singh SA, and Aikawa E. Spatiotemporal Multi-Omics Mapping Generates a Molecular Atlas of the Aortic Valve and Reveals Networks Driving Disease. Circulation 138: 377-393, 2018.

31. Siu SC, and Silversides CK. Bicuspid aortic valve disease. J Am Coll Cardiol 55: 27892800, 2010.

32. Small A, Kiss D, Giri J, Anwaruddin S, Siddiqi H, Guerraty M, Chirinos JA, Ferrari G, and Rader DJ. Biomarkers of Calcific Aortic Valve Disease. Arterioscler Thromb Vasc Biol 37: 623-632, 2017.

33. Steiner I, Krbal L, Rozkos T, Harrer J, and Laco J. Calcific aortic valve stenosis: Immunohistochemical analysis of inflammatory infiltrate. Pathol Res Pract 208: 231-234, 2012. 34. Winchester R, Wiesendanger M, O'Brien W, Zhang HZ, Maurer MS, Gillam LD, Schwartz A, Marboe C, and Stewart AS. Circulating activated and effector memory T cells are associated with calcification and clonal expansions in bicuspid and tricuspid valves of calcific aortic stenosis. J Immunol 187: 1006-1014, 2011. 
722 35. Wu HD, Maurer MS, Friedman RA, Marboe CC, Ruiz-Vazquez EM,

723 Ramakrishnan R, Schwartz A, Tilson MD, Stewart AS, and Winchester R. The lymphocytic

724 infiltration in calcific aortic stenosis predominantly consists of clonally expanded T cells. $J$

725 Immunol 178: 5329-5339, 2007.

726 36. Zhao Y, Nicoll R, He YH, and Henein MY. The effect of statins on valve function and

727 calcification in aortic stenosis: A meta-analysis. Atherosclerosis 246: 318-324, 2016.

728 
729 FIGURE LEGENDS

730 
731 Figure 1. Aortic valve collection summary, bioinformatics pipeline and mapping of sample

732 batches and biological conditions in gene expression profile-based principal component analysis

733 (PCA) plots. (A) Study outline summarizing aortic valve collection. During the study period,

734 aortic valve leaflets were collected from human fetuses at gestational week (week) 9 ( $\mathrm{n}=4$ ), 12-

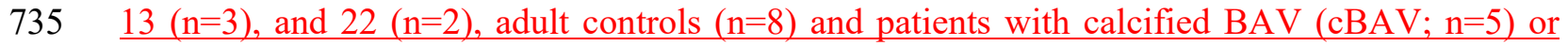

736 TAV (cTAV; n=7). Samples were processed and subject to RNA-seq to characterize their gene

737 expression profiles. Complementary expression data from control, cBAV and cTAV samples

738 was downloaded from GEO (GSE76718). The resulting data set collection was analyzed with a

739 bioinformatics pipeline that included preprocessing of fasta files with cutadapt, alignment

740 against a human transcriptome reference and estimation of gene expression levels with RSEM,

741 and differential expression testing with limma, considering that samples could be ascribed to five

742 experimental batches. Expression profiles were clustered using k-means to obtain a collection of

74350 clusters, which were then annotated with the Canonical Pathway feature of IPA. Twenty-

744 seven clusters associated to at least one canonical pathway (with Benjamini-Hochberg adjusted

745 p-value $<0.05)$ were grouped into six meta-clusters on the basis of concordant annotations.

746 Meta-clusters were finally re-annotated with IPA. (B) Hierarchical clustering of the samples

747 overall gene expression based on Euclidean Distance $(C)$ Gene expression profile PCA plot

748 mapping of biological conditions. Sample points are colored according to fetal developmental

749 stage or adult disease state (no disease, $n=16$; cBAV, $n=15$ and cTAV, $n=16$ ). In PC1, control

750 samples separate from fetal samples and cBAV or cTAV samples. In PC2, early-stage fetal

751 samples (weeks 9 and 13) separate from adult control and disease samples. Percentages on axis

752 labels denote the amount of variance explained by each principal component.

753 
754 Figure 2. Concordance analysis of k-means clustering. K-means-based gene clusters (see

755 Supplementary Fig. S 1B, see https://figshare.com/s/d9a68e0d6faa67e4c6c3) were grouped into

756 metaclusters according to the agreement of their enriched canonical pathways. The white to

757 violet squares in the central heatmap represent the percentage (from 0 to 100) of Canonical

758 Pathways (CP) shared between each pair of $50 \mathrm{k}$-means clusters for which at least one enriched

759 and shared term was found ( $\mathrm{k}=27$ out of the original 50 clusters). Hierarchical clustering of the

760 concordance matrix identified six meta-clusters (MC1-6) The heatmap on the left represents the

761 mean gene expression values for each cluster across biological conditions, on a normalized scale

762 (from -2 to 2, for values below and above the mean, respectively). The dendrogram at the top

763 represents functional relatedness between clusters, which was used to group them into meta-

764 clusters. Meta-clusters are identified with colored boxes (MC1 to MC6, in red, blue, green,

765 purple, orange and yellow, respectively) and represent groups of clusters associated to similar

766 functions.

767 
768 Figure 3. Functional analysis of meta-clusters. The six gene metaclusters (MC1-6) described in

769 Figure 2 were annotated with IPA. Barplots on the right-hand side represent enrichment values

770 for the top five IPA Canonical Pathways associated with each meta-cluster (MC), selected

771 according to enrichment significance and having an adjusted Benjamini-Hochberg $\mathrm{P}<0.05$. Meta-

772 clusters are identified with colored boxes (MC1 to MC6, in red, blue, green, purple, orange and

773 yellow, respectively) and represent groups of clusters associated to similar functions. Central

774 boxes list all the genes associated with the selection of pathways associated with each

775 metacluster. The heatmap on the left represents normalized expression values for individual

776 genes across biological conditions, on a normalized scale (from -2 to 2, for values below and

777 above the mean, respectively).

778 
779 Figure 4. A gene expression signature predictive of CAVD. (A) Correlation of qPCR data (y-

780 axis) vs aortic-valve calcification levels (x-axis) for the 6 selected genes in whole blood (WB).

781 Each point represents an individual's level of gene expression (y-axis) and its aortic valve

782 calcification (x-axis). Dots are colored based on the group to which the individual belongs:

783 PESA-CTL $(\mathrm{n}=52)$, PESA-SD $(\mathrm{n}=52)$ or WB-CAVD $(\mathrm{n}=12)$. Solid lines represent the linear

784 model fit for PESA-SD and WB-CAVD patients. Shaded regions represent the confidence of the

785 linear regression estimate for each calcium level._(B) Receiver operating characteristic (ROC)

786 curve of multivariate logistic regression model predicting CAVD (WB-CAVD) vs preclinical

787 aortic-valve calcification (PESA-SD; thick discontinuous line, AUC=0.97) vs valve calcification

788 (PESA-CTL; thick solid line, AUC=0.91). A third ROC curve for the prediction of valve

789 calcification (PESA-SD) vs no calcification (PESA-CTL; thin solid line, AUC=0.71) is shown.

790 The discontinuous diagonal line indicates a fifty-fifty percent chance to predict each group. (C)

791 Follow-up of the model in PESA subgroups after 3 years. Validation in an independent group of

792 CAVD patients (WB-CAVD, $\mathrm{n}=16$ ) vs a PESA-SD subgroup ( $\mathrm{n}=25$; thick discontinuous line,

793 AUC $=0.90)$ vs a PESA-CTL subgroup ( $n=35$; thick solid line, AUC=0.88). A third ROC curve

794 for the prediction of PESA-SD $(n=25)$ vs PESA-CTL $(n=35$; thin solid line, AUC $=0.71)$ is

795 shown. The thin discontinuous diagonal line indicates a fifty-fifty percent chance to predict each

796 group.

797

798

799 
A

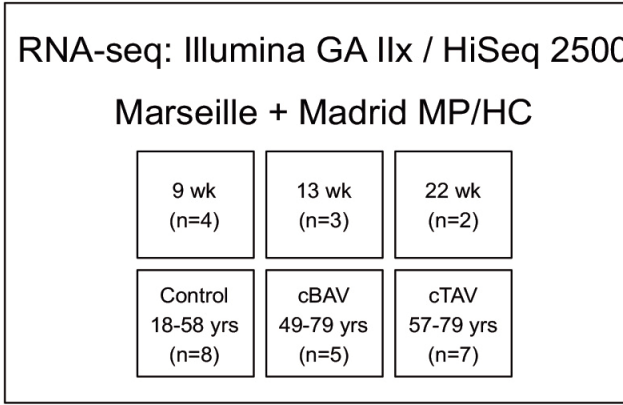

RNA-seq: Illumina HiSeq 2000 GSE76718 (Guauque-Olarte 2016)

\begin{tabular}{|c|c|c|}
\hline $\begin{array}{c}\text { Control } \\
56-68 \text { yrs } \\
(n=8)\end{array}$ & $\begin{array}{c}\text { cBAV } \\
56-68 \text { yrs } \\
(n=10)\end{array}$ & $\begin{array}{c}\text { cTAV } \\
58-71 \text { yrs } \\
(n=9)\end{array}$ \\
\hline
\end{tabular}
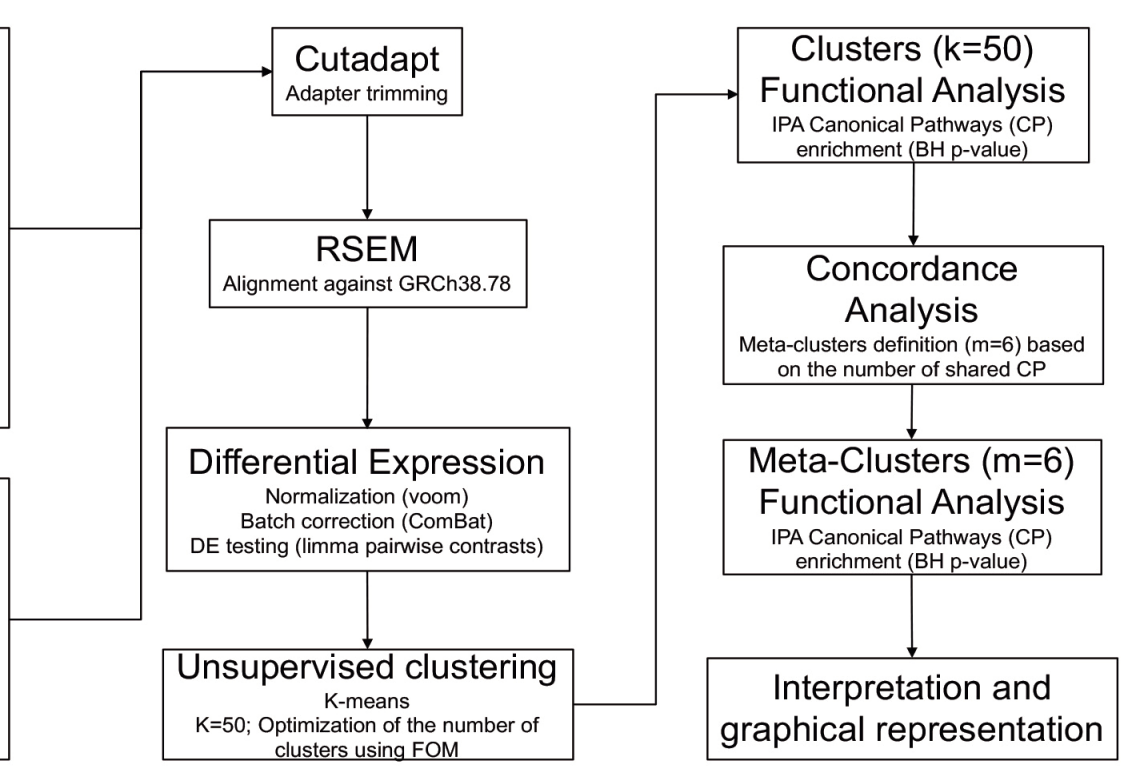

B

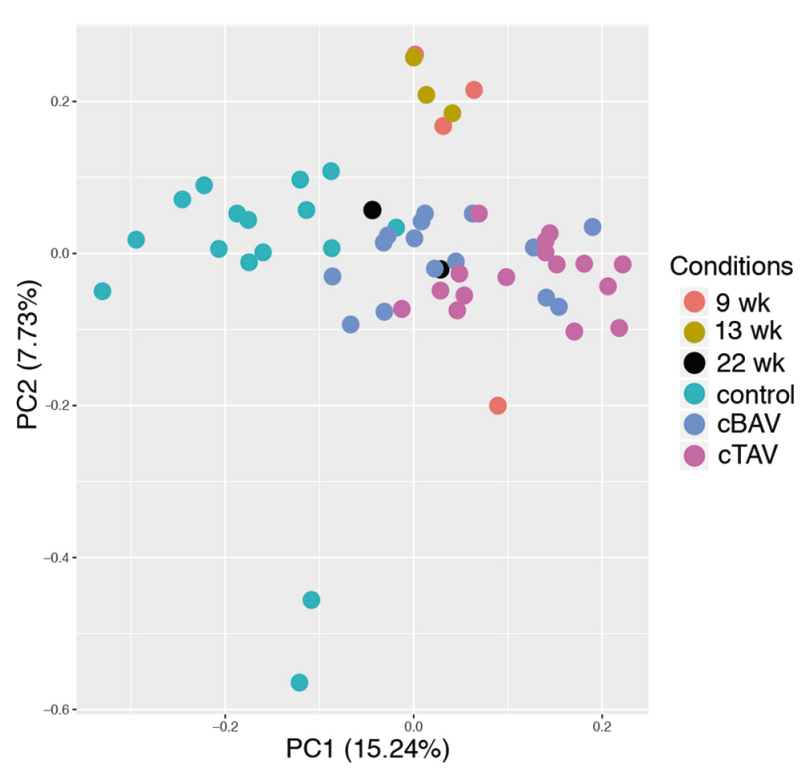

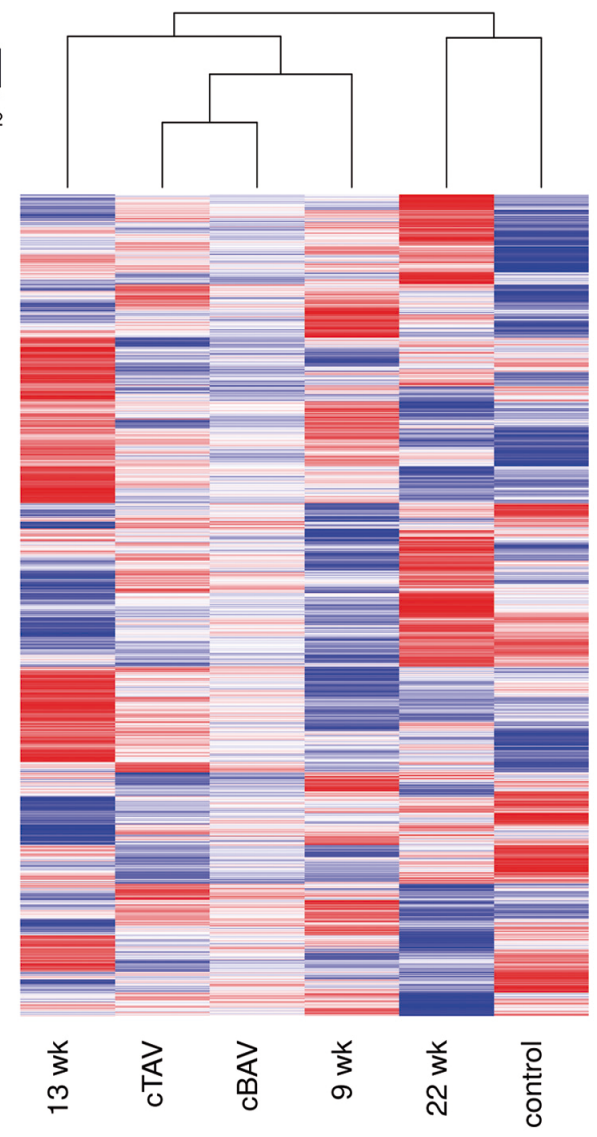

Figure_1_MacGrogan_et_al 


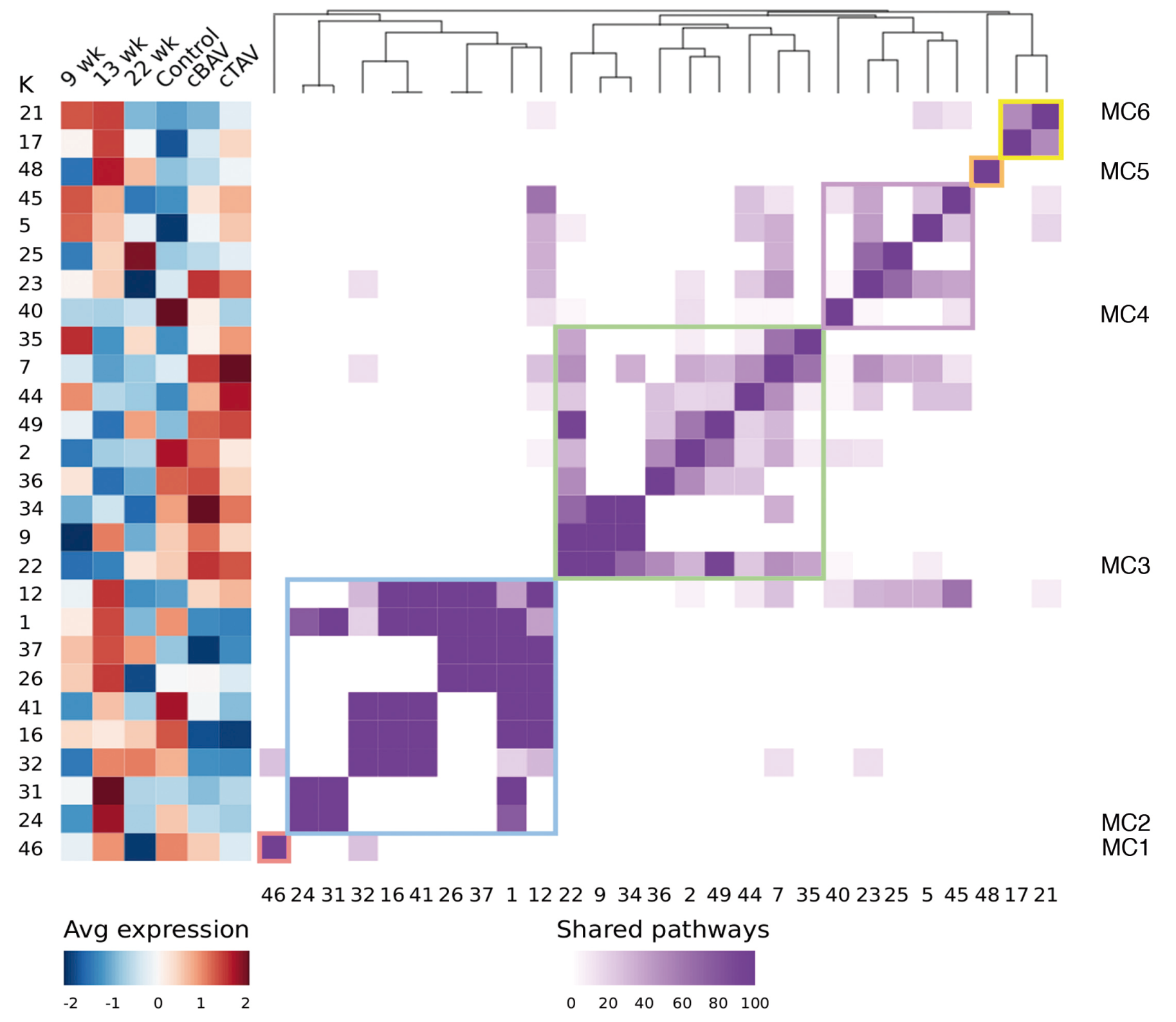

Figure_2_MacGrogan_et_al 

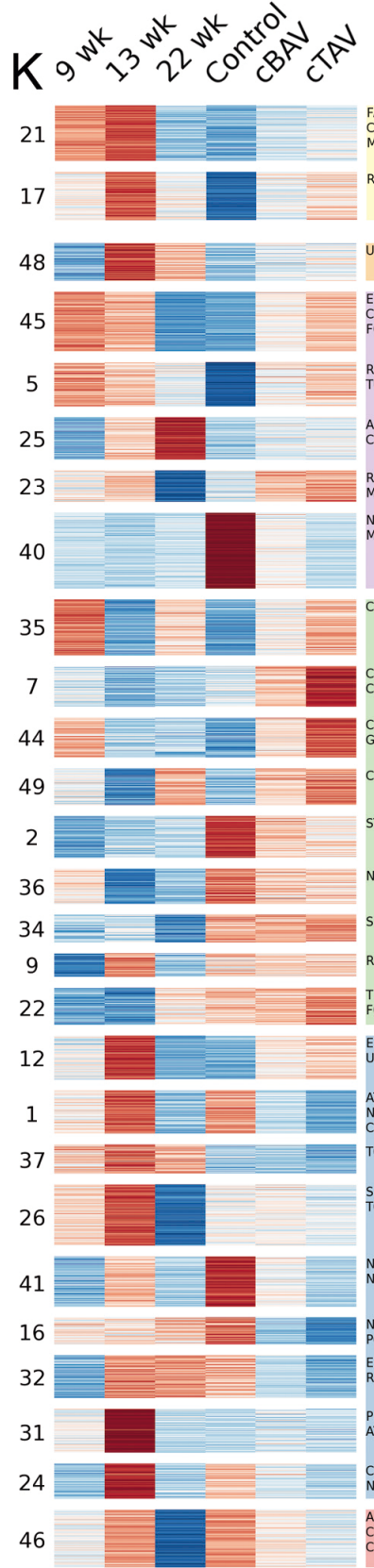

FANCB, HDAC2, SUMO2, MCM2, MSH6, RBBP8, MCM7, SAE1, MSH2, E2F6, FANCG, PCNA, DNMT3A, KDM1A, DNA2, PRKDC, SUMO1, CHEK1,
CDC25C, SMARCD3, RAN, MCMS, RPA3, LIG1, UBA2, RCOR1, CDK2, CDC6, PBRM1, AURKA, CLSPN, YWHAZ, DEF4, PPP2CA, E2F1, CCNB1,

RFC3, TOP2A, CDK1, GDI2, CCNB2, PTPA, RFC4, RNF4, TOP2B, MAP2K4, SENP1, RFC5, RAD1, TRIP12, E2F8, RAD17

BXN4, MBTPS1, MBTPS2, HSPAIL, XBP1, CEBPZ

PHB4, MAPK11, ACVRZA, CDKK, ADAM10, ACTA2, SEMA7A, LIMK1, FZD7, VASP, ARPC1A, RAP2A, CXCL12, SSH1, ACTR3, HIFIA, E2F2,

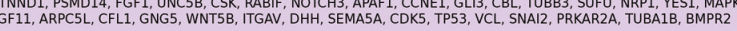

RHOD, APC, ADAMTS12, CDK6, ADAM19, TSPAN5, LINGO1, RALA, EFNB3, ADAMTS16, NLK, ADAMTS5, ENPEP, TTGB3, ARPC5, BCL2L11

ARHGEF9, ROBO2, COPS5, PAPPA2, ADAM22, RASA1, JAK2, PPP3CB, NGEF, ACVR1, SEMA3C, PPP3CA, TGFB3, SOS1, PRKCA, ITGA6, ARHGAP24,
XCR4, ITGA2, IGF1, BIRC2, FRS2, KL

RALB, PDGFB, SHC1, ARHGEF2, ACTB, ROCK1, PLXND1, ITGA3, ARHGEF11, RAPGEF1, GRB2, BAX, PDGFA, TGFB1, ITGB5, SLC9A1, ARHGAP35,

TNA4, RAPGEF3, MAPK3, PSEN2, EPHA6, WNT9A, NFKBIB, MYO7A, ROBO3, DIAPH2, MYL7, MYL5, DPYSL2, IRS2, GSN, EPN2, MYL4, ACTC1,

CD247, RHOH, PLCH2, CCR5, CCL5, NLRC3, IL2RB, MAPK13, CCR4, CD8A, KLRD1, CD3G, PRKCQ, PIK3R6, NLRP12

CD798, IRF8, C5AR1, CXCL8, IGHA1, PIK3R5, IGHM, IGHG1, IGHG4, STAT2, IGHD, CCR7, IKZF1, PIK3CG, IGHG2, SYK, C3AR1, HAVCR2, TREM2,

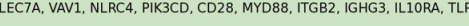

CD1D, JAK3, DLL4, IKBKE, ITGA4, PLCB2, TIMD4, NOTCH4, ILIRN, CCL7, PSEN1, NFKBIE, PLCG2, TREM1, ITGAX, NOD2, RHOF, FCGR1B, TLR1O, GFI1, IL12RB2, TNFSF11, CD79A

CIITA, IL18, PRKCH, TLR8, LAT2, IL12RB1, CR1, FCGR1A, TNFRSF4, TLR7, COL1OA1, CD80, CXCR3

TAT6, PLCD3, MRC1, STAT5A, PLA2R1, IL4R, NFKB1, IKBKG, PIK3CA, LTBR, BHLHE41, IL6R, ICAM1, TNFRSF1A, CCL2, STAT3

NFKBID, APH1B, TLR5, FCGR2B, IL10, IL6, IL2RA

SPI1, PRKCD, IFNGR1, TNFRSF1B, IRAK1, TGFBR2, JAK1, RHOG

RHOA

TNFFF13B, TYROBP, FCER1G, NLRC5, B2M, TLR1, CD86, TLR2, FCGR2A, MSR1, CCR1, CD4, TTGAM, TNFRSF11B, CASP1, TLR4, LGALS9, IKBKB,

EIF2B1, SUV39H1, PPP1CA, PABPC1, CCND1, NDUFA4L2, EIF2AK4, EEF2S2, PPP2R5A, MAPK1, PPID, BACE1, MRAS, LDHA, MAP2K1, NDUFV2,
UOCRFS1. TOMM40L. ACTG2, PGK1, PDPK1, GLS, EIF2AK1, ATPAF1. ATP5PB, EIF2S1

ATP5F1D, NDUFA2, NDUFS6, COX411, COX6A1, COX6B1, HSD17B10, ATPSMG, NDUFS2, NDUFA3, NDUFA12, PPP2R1A, RPL14, RPS13, RPL27A,

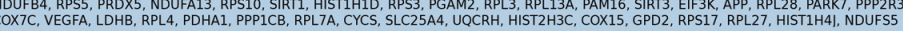
TOMM5, RPS8, NDUFA5, POLR3D, GPX7, RPL15, RPS6, RPS15A, RPS3A, TIMM8A, EIF1AX, PPP2R3A, FGFR2, RPL23

SIRT6, ATG9A, PGAM1, VDAC1, PIK3R2, PPIF, PPP2R5D, ATG4D, MAP2K2, EIF4GG1, EEF3B, EIF3A, GOT2, UQCRC1, GLRX2, XRCC6, MYCN,
TOMM22, TRIM28, NDUFA4, EIF2B3, EIF31, RPS19, SLC25A6, TOMM40, TIMM44, TIMM13 NDUFB8, CYBSR3, MAP1LC3A, RPS9, RPL36, RPL21, UBA52, RPL35, SOD1, FAU, NDUFB7, ATP5ME, RPS12, RPL18A, RRAS, PPP2R5B, BCL2,
NR1H2, RPS16, RPLP2, IRS1, SURF1, COX17, FIS1, RPLP1, RPS15, ATF4, HTRA2, RPL8

NDUFA10, RPS20, RPL35A, RPL7, RPL22, MAPK4, TOMM34, TP53BP1, RPL31, UQCRB, RPL18, RPS26, RPL12, RPL37, RPS25, POLR1E, RPL24,
POLR2F, RPL38 EIF4A3, RPL23A, PFKM, EIF3C, NDUFS4, EIF4A2, GAB1, RPS23, GABARAP, SDHA, AKT2, RPS21, RPL32, RPL29, PAIP2, RPS27A, RPS29, RPL13,
RPL37A, RPL10, RPL41, GABARAPL2, EIF2AK3, RPL30, PPARGC1A, COX7A1, RPS11

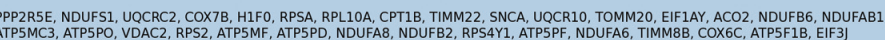

COX5B, NDUFV1, RAF1, FGFR1, COX8A, COX7A2, NDUFB1, ATPSMC1, CYC1, TOMMG, NDUFB11, HSPA5, NDUFA1, APH1A, SDHB, SDHD, DUAA, UQCR11, OGDH, NDUFB10, NDUFA11, UQCRQ, PPP2CB, TXN2

ARAF, AKT1S1, GNA12, SMPD1, GNA12, ASAH1, RAC1, IDUA, RPTOR, SIPR2, SMPD4, MLST8, CSNK1E, MAPR7, FGFRL1, RHOT2, GNB2, MAPK9,
CAPNS1, PSENEN, NCSTN, S1PR3, HDAC5, AP2A2, HYAL2, MAP2K7, CAPN1, AKT1, HRAS, ADCY9, S1PR1, HEXDC, CAPN10, GNA11, DCTN1, CLTB, POLR2E, GPAA1
Avg expression

$\begin{array}{lllll}-2 & -1 & 0 & 1 & 2\end{array}$
MC Top 5 significant pathways

Role of CHK Proteins in Cell Cycle Checkpoint Control Sumoylation Pathway

6 Cell Cycle Control of Chromosomal Replication Cell Cycle: G2/M DNA Damage Checkpoint Regulation Role of BRCA1 in DNA Damage Response

5 Unfolded protein response

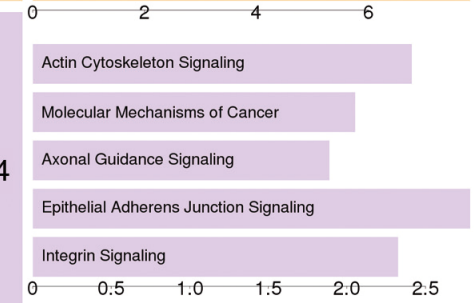

Th1 and Th2 Activation Pathway

TREM1 Signaling

3

Communication between Innate and Adaptive Immune Cells

Phagosome Formation

Dendritic Cell Maturation

$\begin{array}{llllllll}0 & 0.5 & 1.0 & 1.5 & 2.0 & 2.5 & 3.0 & 3.5\end{array}$

Mitochondrial Dysfunction

EIF2 Signaling

2

Oxidative Phosphorylation

Sirtuin Signaling Pathway

Regulation of elF4 and p70S6K Signaling

$\begin{array}{lllll}0 & 1 & 2 & 3 & 4\end{array}$

1 Sphingosine-1-phosphate Signaling

Endocannabinoid Developing Neuron Pathway

Amyloid Processing

Huntington's Disease Signaling

Dermatan Sulfate Degradation (Metazoa)

$\begin{array}{llllll}0 & 2 & 4 & 6 & 8 & 10\end{array}$

Enrichment

\section{Figure_3_MacGrogan_et_al}


A

ASSC3

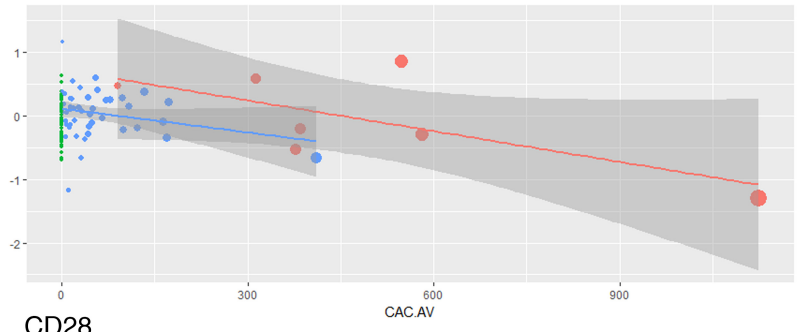

CD28

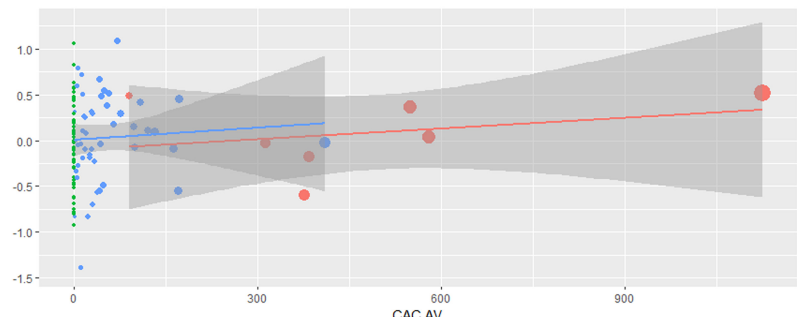

ITK

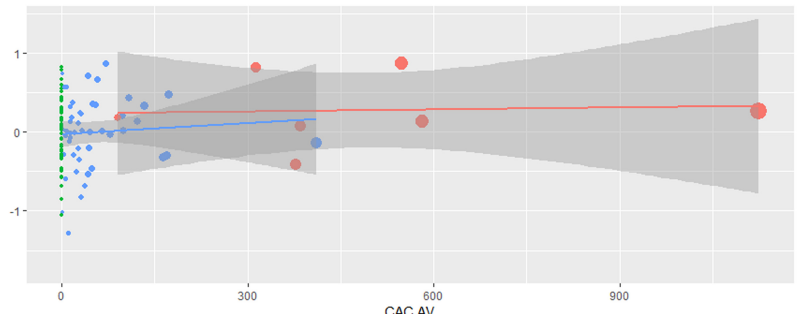

B

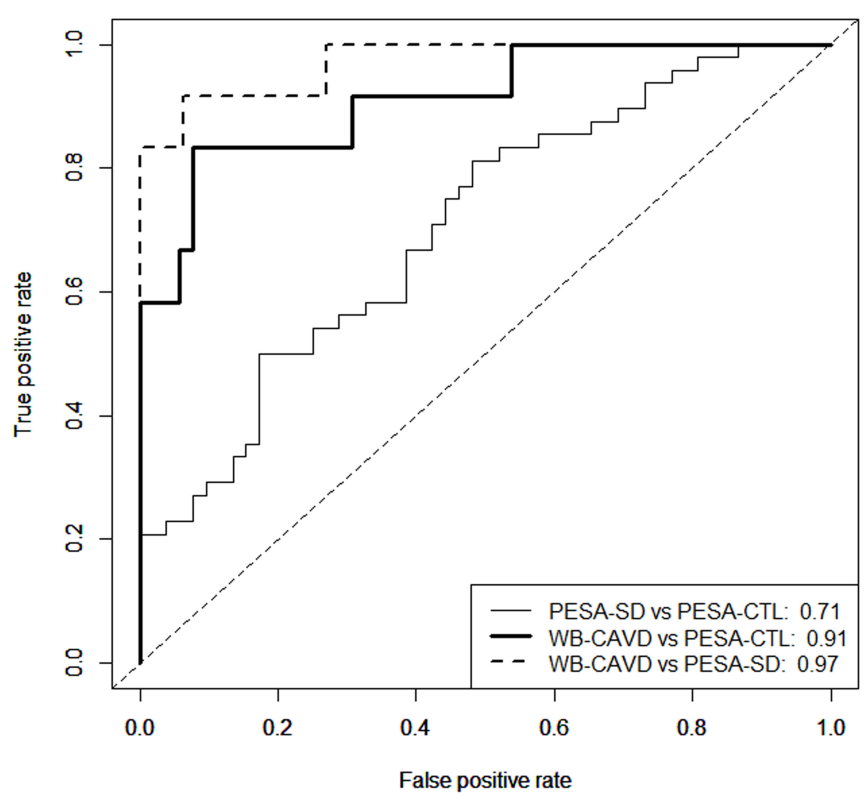

LINS1

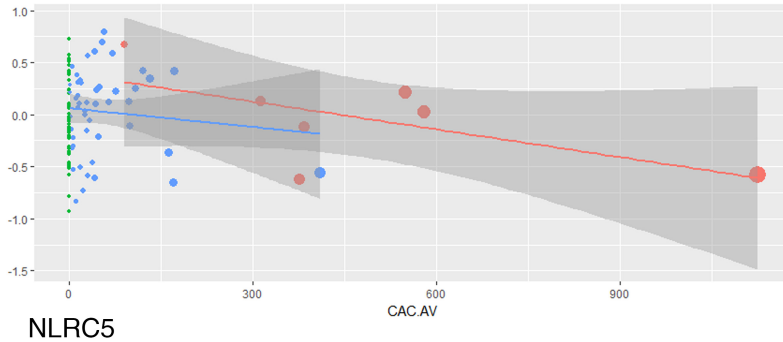

CAC.AV

- 0

- 300

- 600

Condition

- WB-CAVD

- PESA-CTL

- PESA-SD

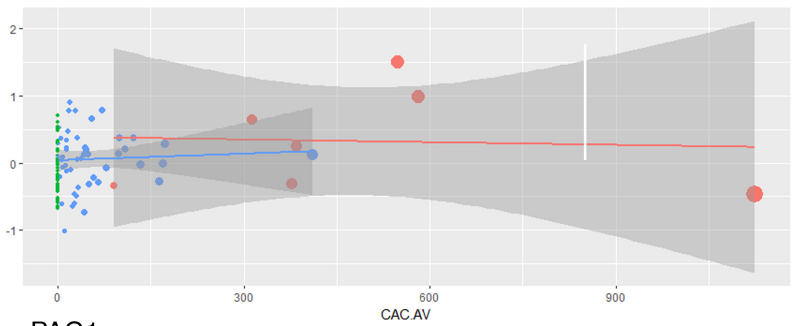

PAG

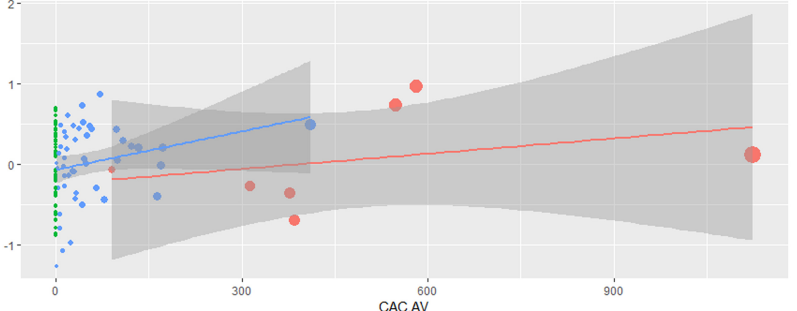

C

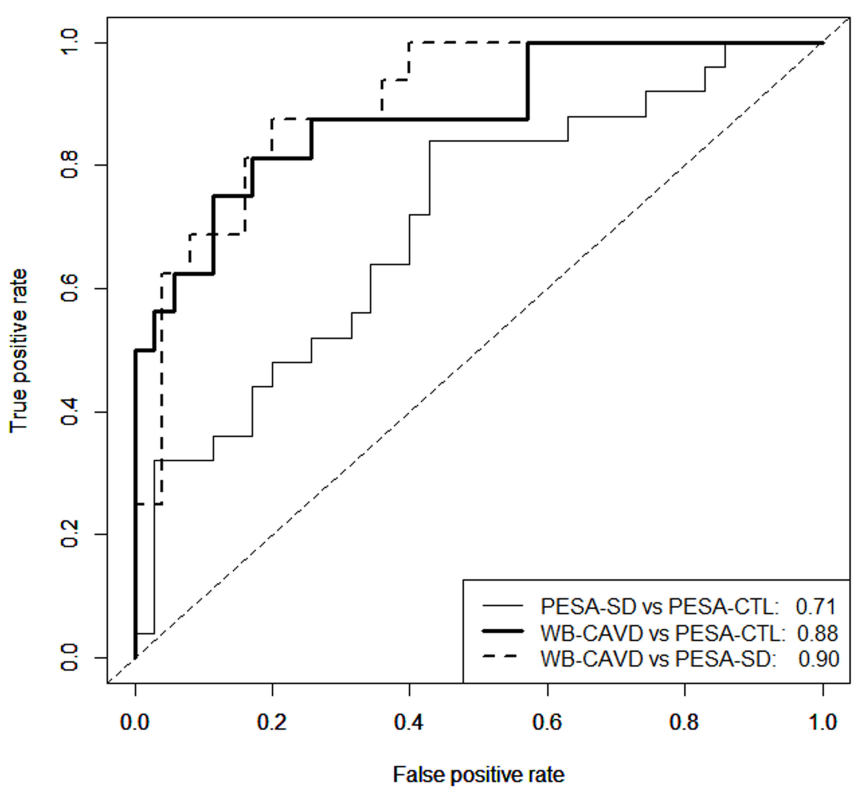

Figure 4_MacGrogan et al 